\title{
Hiperplasia papilar endotelial intravascular
}

\section{Intravascular papillary endothelial hyperplasia}

\author{
Fernández García-Guilarte, R.*, Enríquez de Salamanca Celada, J .**, Comenero, I.***
}

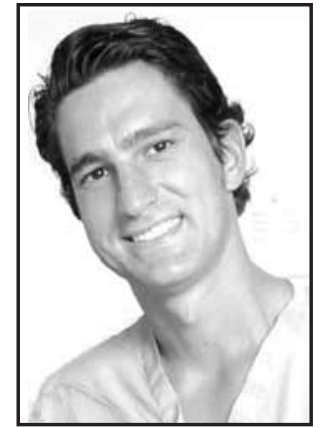

García-Guilarte, R.

\section{Resumen}

La hiperplasia endotelial papilar intravascular (PEH), también conocida como hemangioendotelioma vegetante intravascular o pseudoangiosarcoma de Masson, es una lesión vascular benigna inusual, no neoplásica, que consiste en una rara proliferación endotelial reactiva, comúnmente localizada en la piel y en los tejidos subcutáneos. Es importante remarcar los aspectos que distinguen esta entidad de otras lesiones neoplásicas para evitar tratamientos inapropiados: se diferencia del angiosarcoma por su circunscripción, la localización en un vaso, su asociación con trombosis y su arquitectura papilar sin atipia citológica significativa o áreas de crecimiento sólido. El tratamiento consiste en la completa resección de la lesión incluyendo amplios márgenes para evitar la recurrencia.
The intravascular papillary endothelial hyperplasia $(\mathrm{PEH})$, also known as hémangioendothéliome végétant intra-vasculaire or Masson's pseudoangiosarco$\mathrm{ma}$, is an unusual benign, non-neoplastic, vascular lesion, with rare non-neoplasic reactive endothelial proliferation most commonly located in the skin or subcutaneous tissues. It is important to remark the features that can distinguish this entity from other neoplasms to avoid inappropriate treatment. Pathological characteristics that distinguish $\mathrm{PEH}$ from angiosarcoma could be reduced to circumscription of the lesion, location in a vessel or association with thrombus and papillary architecture without significant cytologic atypia or areas of solid growth. Treatment consists of complete resection of the tumor, including wide enough margins to avoid recurrence.

\author{
Palabras clave Hiperplasia Endotelial Papilar Intravas- \\ cular, Hemangioendotelioma Vegetante \\ Intravascular, Tumor de Masson, Mal- \\ formación Vascular \\ Código numérico 173
}

\begin{tabular}{|ll} 
Key words & Intravascular Papillary Endothelial \\
& Hyperplasia, Vegetans Intravascular \\
& Hemangioendothelioma, Masson's \\
& tumor, Vascular malformation \\
Numeral Code & 173
\end{tabular}




\section{Introducción}

La hiperplasia papilar endotelial intravascular (PEH) también conocida como hemangioendotelioma vegetante intravascular o pseudoangiosarcoma de Masson, es una lesión vascular benigna inusual, noneoplásica. Fue descrita por Pierre Masson en 1923 como una neoplasia peculiar en un varón de 68 años con una hemorroide dolorosa y ulcerada que no podía reducirse. La hemorroide fue escindida y se determinó que histológicamente se caracterizaba por una proliferación papilar endotelial atípica que mimetizaba al angiosarcoma (1).

La PEH es una rara proliferación no neoplásica reactiva endotelial, comúnmente localizada en la piel y tejidos subcutáneos a pesar de haberse descrito en múltiples localizaciones del cuerpo humano.

Presentamos 5 casos de PEH, 4 de ellos en la mano (Fig. 1) y 1 en el pie (Fig. 2). Es importante remarcar los aspectos que ayuden a distinguir esta entidad de otras neoplasias, con el objetivo de evitar tratamientos inapropiados; por ello, en todos los casos, el diagnóstico fue en un primer tiempo clínico, por sus características de presentación y posteriormente anatomopatológico, de confirmación.

\section{CASOS CLINICOS}

Durante 11, años en el Servicio de Patología Quirúrgica del Hospital Niño Jesús (Madrid, España), tan solo 5 casos cumplieron los criterios diagnósticos del tumor descrito por Masson. Revisaremos sus características clínicas y las secciones teñidas con Hematoxilina-Eosina, así como las tinciones de fibras elásticas según técnica de Van Gieson (Tabla I). Todos los casos tuvieron lugar en mujeres de raza blanca, en el intervalo de edad de 9 a 47 años (alguno de los pacientes provienen de la práctica privada de uno de

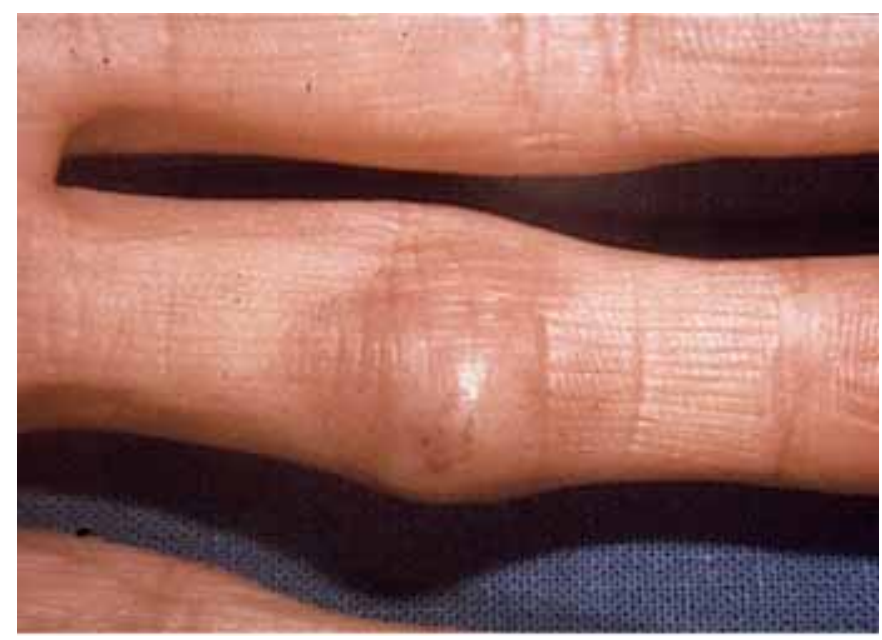

Fig. 1. Afectación del aspecto volar de la articulación interfalángica proximal en paciente de $\mathbf{4 7}$ años de edad. los autores del trabajo, de ahí que el rango de edad exceda la propia de la casuística habitual de un hospital infantil).

El comportamiento clínico fue similar en todos los casos, apareciendo una masa con márgenes poco claros y coloración púrpura, de crecimiento progresivo. Tan solo 2 de las lesiones feueron dolorosas a la palpación y sólo 2 pacientes relacionaron la presentación inicial del tumor con traumatismos previos (Fig. 1,2).

La cirugía consistió en la completa resección de las lesiones con $2 \mathrm{~mm}$ de margen, seguida de cierre directo. Hubo 1 caso de dehiscencia menor, coincidiendo con el único caso de afectación de la planta del pie. Dos de las lesiones recurrieron y fueron de nuevo resecadas con márgenes mayores, sin nuevas recidivas.

\section{Discusión}

Existen muy pocos artículos sobre la PEH en la literatura. La familiarización con esta entidad es importante para el diagnóstico diferencial frente a tumores vasculares. La PEH representa un proceso vascular benigno sin ningún aspecto clínico específico.

Las características patológicas que podrían ayudar a distinguir la PEH del angiosarcoma podrían reducirse a la circunscripción de la lesión la localización en un vaso o su asociación con trombosis, así como su arquitectura papilar sin atipia citológica significativa o áreas de crecimiento sólido. El reconocimiento de los hallazgos morfológicos de esta lesión y su inclusión en el diagnóstico diferencial de tumores vasculares, puede reducir la tendencia al diagnóstico equivocado de esta entidad como angiosarcoma y evitar así un tratamiento agresivo innecesario (2).

Típicamente, estas papilas están alineadas en una sola capa de células endoteliales hinchadas alrededor de un tronco de colágeno. No hay ninguna papila mul-

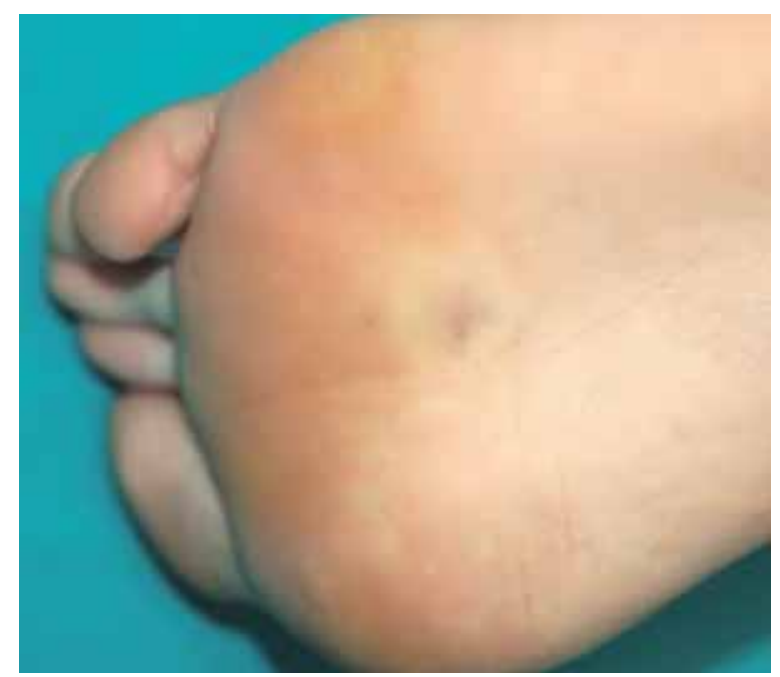

Fig. 2: Afectación de la planta del pie en paciente de 17 años de edad. 
Tabla I. Hallazgos clínicos

\begin{tabular}{|c|c|c|c|c|c|}
\hline & Paciente 1 & Paciente 2 & Paciente 3 & Paciente 4 & Paciente 5 \\
\hline Edad (años) & 13 & 9 & 47 & 24 & 17 \\
\hline Localización & Mediopalmar & Hipotenar & $4^{\circ}$ Dedo & Mediopalmar & Planta del pie \\
\hline Duración (Meses) & 12 & 12 & 3 & 6 & 4 \\
\hline Tamaño $(\mathrm{cm})$ & 0.7 & 1 & 0.8 & 0.6 & 1.2 \\
\hline Clínica & Masa blanda dolorosa & Nódulo firme violáceo & Nódulo firme rojizo & Nódulo indurado & Nódulo firme violáceo \\
\hline Tratamiento & $\begin{array}{l}\text { Resección completa } \\
\text { con } 2 \mathrm{~mm} \text { de margen }\end{array}$ & $\begin{array}{l}\text { Resección completa } \\
\text { con } 2 \mathrm{~mm} \text { de margen }\end{array}$ & $\begin{array}{l}\text { Resección completa } \\
\text { con } 2 \mathrm{~mm} \text { de margen }\end{array}$ & $\begin{array}{l}\text { Resección completa } \\
\text { con } 2 \mathrm{~mm} \text { de margen }\end{array}$ & $\begin{array}{l}\text { Resección completa } \\
\text { con } 2 \mathrm{~mm} \text { de margen }\end{array}$ \\
\hline Recurrencia Local & - & $\begin{array}{l}\text { Tras } 8 \text { meses } \\
\text { de la cirugía }\end{array}$ & $\begin{array}{l}\text { Tras un mes } \\
\text { de la cirugía }\end{array}$ & - & - \\
\hline Seguimiento & 6 meses & 2 años & 5 años & 5 años & 3 meses \\
\hline
\end{tabular}

ticapa, áreas sólidas, necrosis y poca o ninguna atipia. Hay poca evidencia de mitosis. En los estadios iniciales, las papilas están compuestas de fibrina; en los estadios finales existe agrupación y fusión de la papilas formándose una red anastomótica de vasos sanguíneos dentro de un entramado de tejido conectivo (Fig. 3).

Desde la lámina interna de las grandes venas nacen papilas, cubiertas por endotelio. Estas papilas aumentan en tamaño dando lugar a proyecciones polipoides (vegetaciones) cuyos extremos poseen capilares y fibrina. Estas papilas tienden a cubrir progresivamente el lumen de las venas. A medida que aumentan, estas ramificaciones endoteliofibrinosas se acercan y contactan entre ellas, gradualmente rellenando la vena y obstruyéndola. Como consecuencia, la sangre no puede circular y finalmente se forma un trombo (3) (Fig. 4).

La PHE puede aparecer como una forma primaria o pura, desarrollada en un vaso distendido o puede asociarse a otras lesiones vasculares como hemangiomas, granulomas piógenos (4) o malformaciones linfáticas, pero la causa de esta condición es desconocida. Probablemente haya factores hormonales que influyan en

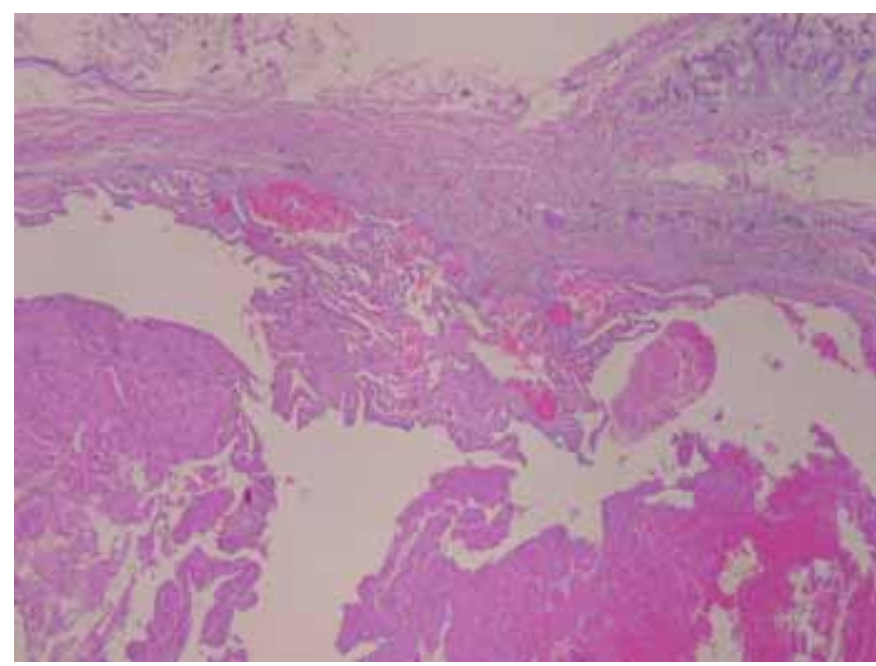

Fig. 3. Anatomía Patológica mediante tinción con Hematoxilina-Eosina y microscopía óptica, 40X: se puede observar cómo las papilas endoteliales nacen desde el endotelio. su histogénesis $(5,6)$ dada su preponderancia femenina al igual que en otras tumoraciones vasculares benignas, tal y como nosotros vemos en nuestra serie en la que todos los casos ocurrieron en mujeres. Además, hay quien describe esta entidad como un proceso reactivo por un trombo dada la frecuente historia de traumatismo previo.

La presentación clínica es variable; puede aparecer en casi todo el cuerpo humano (7). La localización más frecuente es en la mano; por ello, los cirujanos de la mano deben tener conocimiento y tener presente este raro tumor para considerarlo en el diagnóstico diferencial con los tumores vasculares de la mano, puesto que la PEH puede curarse mediante la completa resección quirúrgica evitando un tratamiento inapropiadamente agresivo $(8,9)$.

La principal característica de la PEH es su gran parecido, tanto clínico como histológico, con el sarcoma de tejidos blandos y su posible malinterpretación como tal. Por tanto, debe hacerse énfasis en el estudio clínico, radiológico e histológico de la misma (10).

Su tratamiento consiste en la completa resección de la tumoración, incluyéndo márgenes amplios

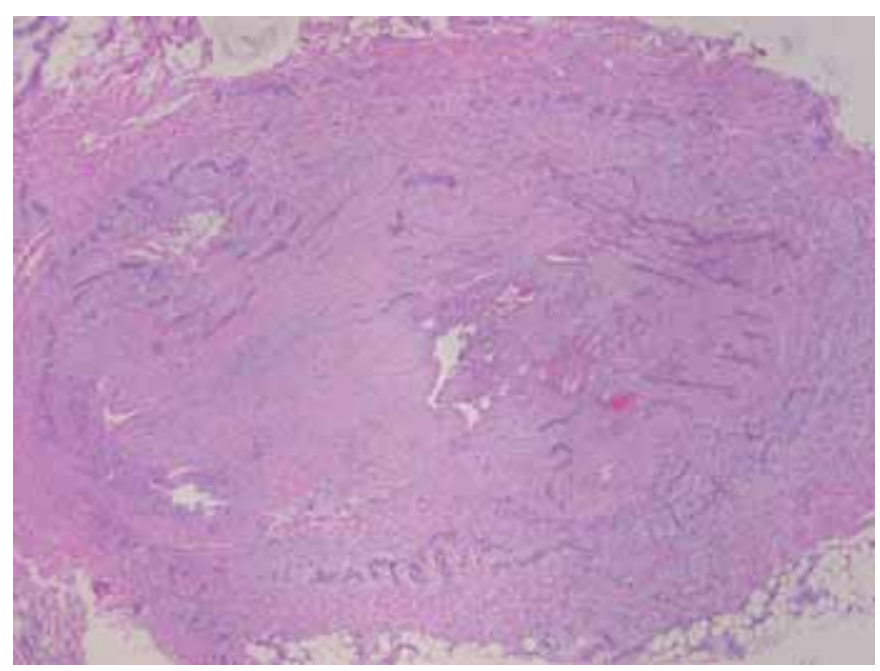

Fig. 4. Anatomía Patológica mediante tinción con Hematoxilina-Eosina y microscopía óptica 40X: se puede observar cómo en un estadio final, la vena se rellena por ramificaciones endotelio-fibrinosas. 
para evitar así su recurrencia. Basándonos en el carácter benigno y circunscrito de esta lesión, es suficiente la escisión con $2 \mathrm{~mm}$ de margen en los casos en que sus límites son evidentes, pero pueden requerirse márgenes mayores en caso de no ser así. Es importante añadir que no existe consenso acerca de los márgenes de resección. No obstante, dado que el tamaño del tumor es generalmente pequeño, suele ser posible el cierre directo tras la extirpación (11).

\section{Conclusiones}

La hiperplasia papilar endotelial intravascular o tumor de Masson, es una lesión vascular benigna no neoplásica, no habitual. Es importante no despreciarla a la hora de realizar el diagnóstico diferencial frente a otras tumoraciones vasculares dada su frecuente malinterpretación con el angiosarcoma. Su localización más frecuente se da a nivel de las manos, por lo que los cirujanos de la mano deben tenerla siempre presente. Para su diagnóstico, el estudio es fundamentalmente anatomopatológico. Una vez cumplidos los criterios diagnósticos que la identifican, la extirpación con márgenes suele ser suficiente.

\section{Agradecimiento}

A la Dra. Beatriz Berenguer por su gran colaboración en la redacción de este artículo, a pesar de no haber participado quirúrgicamente en los casos descritos.

\section{Dirección del autor}

Dr. Rubén Fernández García-Guilarte

C/ Pedro Rico, $19-10^{\circ} \mathrm{D}$

Madrid - 28029 (Madrid)

e-mail:rubenfergar@gmail.com

\section{Bibliografía}

1. Steffen C.: "The man behind the eponym C.L. Pierre Masson" The American Journal of Dermapathology, 2003;25(1):71.

2. Branton PA, Lininger R, Tavassoli FA. "Papillary endothelial hyperplasia of the breast: the great impostor for angiosarcoma: a clinicopathologic review of 17 cases". Int J Surg Pathol. 2003; 11(2):83.

3. Masson P.: "Hémangioendothélioma végétant intra-vasculaire". Bulletin Société Anatomique 1923;93:517.

4. González López MA, Alija Senra A, Bousa Montero, Hidalgo Garcia Y.: "Hiperplasia endotelial papilífera intravascular en el seno de un granuloma piógeno". Rev Intern Dermatol Dermocos 2002; 5:461.

5. Clearkin CK, Enzinger FM.: "Intravascular papillary endothelial hyperplasia”. Arch Pathol Med 1976(100): 441.

6. Hashimoto H, Daimaru Y, Enjoji M.: "Intravascular papillary endothelial hyperplasia: a clinicopathologic study of 91 cases". Am J Dermatol 1983 (5): 539.

7. Weber FL, Babel J.: "Intravascular papillary endotelial hyperplasia of the orbit". B. J.of Ophthal., 1981; 65: 18.

8. Taricco MA, Vieira JO Jr, Machado AG, Ito FY.: "Intravascular papillary endotelial hyperplasia causing cauda equina compression: case report". Neurosurgery 1999; 45(6):1478.

9. Schwartz IS, Parris A.: "Cutaneous intravascular papillary endotelial hyperplasia: a benign lesion that may simulate angiosarcoma". Cutis 1982; 29(1):66.

10. Pantanowitz L, Muc R, Spanger M, Sonnendecker H, McIntossh WA.: "Intravascular papillary endothelial hyperplasia (Masson's tumor) manifesting as a lateral neck mass". Ear Nose Throat J. 2000;79(10):806.

11. Berenguer B, Enríquez de Salamanca Celada, González B, Rodríguez P, González I.: "Intravascular papillary endotelial hyperplasia: report of four cases in the hand". Eur J Plast Surg 2002 (25):280. 